\title{
Vulnerability Analysis of Urban Rail Transit Network by Considering the Station Track Layout and Passenger Behavior
}

\author{
Taoyuan Yang $\mathbb{D},{ }^{1}$ Peng Zhao $\mathbb{D}^{1},{ }^{1}$ Ke Qiao $\mathbb{D}^{2},{ }^{2}$ Xiangming Yao $\mathbb{D}^{1},{ }^{1}$ and Tao Wang $\mathbb{D}^{1}$ \\ ${ }^{1}$ School of Traffic and Transportation, Beijing Jiaotong University, Beijing 100044, China \\ ${ }^{2}$ State Research Center of Rail Transit Training and Accreditation, Beijing Jiaotong University, Beijing 100044, China \\ Correspondence should be addressed to Ke Qiao; qiaoke@bjtu.edu.cn
}

Received 25 October 2019; Revised 27 May 2020; Accepted 16 November 2021; Published 14 December 2021

Academic Editor: Shamsunnahar Yasmin

Copyright (C) 2021 Taoyuan Yang et al. This is an open access article distributed under the Creative Commons Attribution License, which permits unrestricted use, distribution, and reproduction in any medium, provided the original work is properly cited.

\begin{abstract}
The vulnerability of an urban rail transit (URT) network is an index that reflects its ability to cope with risks. However, existing URT network vulnerability studies have paid less attention to station track layout and passenger choice behavior, both of which significantly affect the consequences of a disruption incident. In the present study, we first analyze an actual scenario of URT section disruption and passenger behavior during an incident. Then, we propose two section vulnerability indexes that quantitatively evaluate the effect of a URT section disruption from two aspects: detour delay and loss in passenger flow. To make the application scenario of this method more realistic, the track layout and depot location are taken into account. By considering the relationship between train routing and the sections, a concept of "dominant section" is put forward to make the calculation of the vulnerability indexes more efficient and can be used for a simultaneous multi-section-disruption scenario. Finally, a case study of the Beijing Subway network is provided. The results show that disruptions in only a few critical sections can significantly affect the URT network passenger flow. Disruption of only $3 \%$ of the sections can lead to $80 \%$ passenger-flow loss, which reflects the high vulnerability of URT networks. The method proposed in this paper can provide support for the evaluation of URT network performance.
\end{abstract}

\section{Introduction}

The urban rail transit (URT) system has gradually become the backbone strength of urban traffic owing to its fast, punctual, and large-capacity characteristics. However, a URT system is more vulnerable than the urban road transportation system [1]. One reason is that the train routes of a URT system are generally single. Therefore, once disruption occurs, bypassing the trains is difficult. Furthermore, the delay of one train will "knock on" to the others. Another reason is that the URT network is more sparse than the urban road network. Passengers in the URT system have fewer detour routes during a disruption, which seriously affects the accessibility of passengers. With the frequent disruptions in URT systems, identifying the critical components of the URT network and guaranteeing the security and stability of the system are important.
Vulnerability is an index that measures the degree of system-capability loss in disaster events [2]. For the road transportation system, Berdica et al. [3] defined vulnerability as "susceptibility to incidents that can result in considerable reductions in road-network serviceability." This definition is equally valid for other modes of transportation such as the URT system. The component whose disruption causes the greatest reduction in the network capability is considered the critical component. By identifying the critical component or combination of critical components, we can analyze the network vulnerability.

In recent years, numerous studies on transportation network vulnerability have been conducted. According to these methods, research on transportation network vulnerability can be divided into two categories: network-topology-based and transportation supply-demand relationship-based methods. The network-topology-based 
methods consider a transportation network as a set of nodes and edges using the complex-network theory and use static indexes such as node degree, node betweenness, and clustering coefficient to measure the effect of a node or edge disruption on the network performance $[4,5]$. These static index-based methods are easy to implement, but they ignore the dynamic distribution of traffic flow in a transportation network. For example, one edge of a transportation network may not be critical in terms of topology. However, once it carries a large amount of traffic flow, which may be a critical edge in the network. The transportation supply-demand relationship-based methods consider the interaction between the transportation network and travel demand and measure the network vulnerability by analyzing the redistribution of traffic flow after disruption events. Embedding a user equilibrium model into the network vulnerability analysis is a common method of traffic flow redistribution $[6,7]$. However, because of the computational complexity of solving a large-scale network-equilibrium problem, applying this method to simultaneous multi-segment-disruption scenarios is difficult. The research of Wang et al. [8] and Xu et al. [9] demonstrated that the effect of simultaneous multisegment disruption is not a simple superposition of each single-segment disruption. Nevertheless, in their researches, the traveler behavior is not fully considered for the sake of calculation simplicity. Furthermore, the URT system is different from the road transportation system. Limited by the turn-back condition of the railway line, the disruption of a single section may cause secondary disruption of the adjacent sections. To solve this problem, some research works analyzed the effect of partial delay or disruption of an entire line on the network passenger flow [10, 11], whereas most of the other studies continued to use the vulnerability assessment methods of road transportation, which are inaccurate for the characterization of the URT system [1].

In summary, some deficiencies remain in existing URT network-vulnerability research in terms of disruption-scenario characterization and passenger-behavior description. Focusing on the section-disruption scenario of a URT system, the present work first analyzes the effect of station track layout and depot location on the turn-back condition and then presents a passenger behavior model for a sectiondisruption scenario. On this basis, two vulnerability indexes considering passenger behavior are provided. To calculate the vulnerability indexes in a simultaneous multi-sectiondisruption scenario, we propose an algorithm based on the correlation between the train routing, travel demand, and rail section. Finally, a case study of the Beijing Subway network is carried out. The critical sections or section combinations are identified by calculating the vulnerability indexes. Subsequently, a vulnerability analysis of the network is presented.

\section{URT Section-Disruption Scenario and Passenger Behavior}

In this paper, we consider the disruption on a component of a URT system. The component could be a section, a station, or even a hub. When the disruption occurs on a station or a hub, all the sections connecting to the station or the hub turns to be disrupted. Finally, any disruption on the URT system could be represented as disruption on sections. We focus on the section-disruption scenario in this paper.

In contrast to a road network, when a single section of a URT system is disrupted due to equipment failure or manmade attacks, secondary disruptions in the adjacent sections usually follow because of the lack of tracks for overtaking or turning back. In addition, the URT network is sparser than the road network. Therefore, passengers may abandon the URT system after a disruption occurs if no detour route is available or the detour route is very long. This behavior should be considered in the vulnerability analysis.

2.1. URT Section-Disruption Scenario. According to the severity of the incident, the disruption incidents can be divided into three categories: short-term section disruption, long-term section disruption, and disruption of the entire line/network. Short-term section disruption refers to an incident in which a single section is disrupted for less than 15 min (for some busy lines with a quite short headway, this time may even be shorter). Long-term section disruption usually lasts more than $15 \mathrm{~min}$, and disruption of the entire line/network means that the operation of the line/network completely breaks down due to serious accidents. The operation in a short-term section disruption can be quickly resumed, which does not affect passenger accessibility. The probability of an entire line/network disruption is relatively small, and such incidents are usually caused by serious largescale faults that are less correlated with a single section. Therefore, this paper mainly focuses on the long-term section disruption, which frequently occurs in daily operations and greatly affects passenger accessibility. In addition, because of the different locations and passenger flow, the effect of each section disruption on the URT network is quite different. A critical section is a section whose disruption may cause serious consequences. Identification of the sections that greatly influence the network is very important for the analysis and improvement of network performance.

When a long-term disruption (more than $15 \mathrm{~min}$ ) occurs in a URT section, the operator usually operates short-turn trains at both sides of the fault section to maintain operation as far as possible. However, because of the limited station track layout, not all stations can perform turn-back operations of the trains, and some stations perform turn-back operations on only one side. Figure 1 shows several common turn-back modes, and Figures 1(a) and 1(b) show the unilateral and bilateral turn-back operations, respectively, using the turn-back track. The crossover track can also perform a turn-back operation, but it is seldom used in daily operations because the turn-back operation that uses the crossover track must occupy the mainline. When a section breaks down, if the operation at one side of the crossover track is disrupted, trains at the other side of the crossover track could use the mainline and the crossover track to turn back. Figures 1(c) and 1(d) show that the crossover track can perform turn-back operations for trains from both directions in case of emergency. 


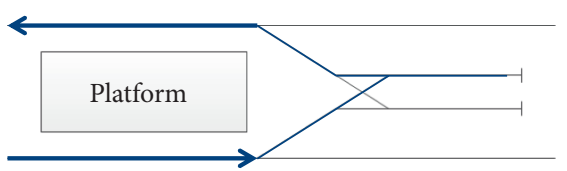

(a)

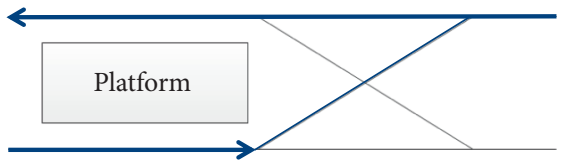

(c)

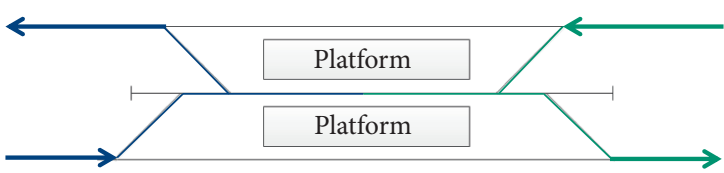

(b)

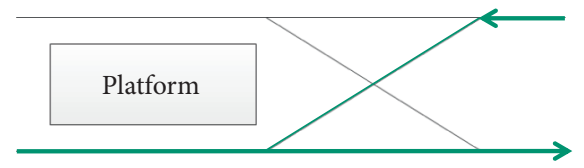

(d)

FIGURE 1: Turn-back modes of URT trains: (a) unilateral turn-back operation using the turn-back track, (b) bilateral turn-back operation using the turn-back track, (c) station-front turn-back operation using the crossover using track, and (d) station-behind turn-back operation the crossover track.

The sections that operate short-turn trains require two conditions: first, the two stations at the two ends of these sections need tracks for the turn-back operation, and second, these sections need tracks that are connected to the depot for trains that enter or leave the depot. Figure 2 shows that if the section between Yonganli and Guomao is disrupted, trains in the two directions can turn back at Xidan and Guomao, respectively. In addition, two depots between the Pingguoyuan-Xidan and Guomao-Sihui sections are available. Thus, short-turn operations can be performed in these sections. In this case, the Yonganli-Guomao section is the primary disrupted section, and the sections between Yonganli and Guomao are the secondary disrupted sections. Although the Wangfujing station can perform turn-back operations, only the trains that run in the downstream direction can turn back at Wangfujing. Therefore, the section between Xidan and Wangfujing is also disrupted. Thus, the turn-back direction of the turn-back station is clearly an important factor that affects the disruption consequence.

2.2. URT Passenger Behavior during Disruption. Because of the high density of the road network and abundant detour routes, existing research on road-network vulnerability usually assumes that the travel demand remains unchanged. However, during a URT disruption, passengers may choose other transportation modes besides taking a detour route from the URT system. In section-disruption scenario, the time required for recovery is much longer compared with the travel time of URT passengers. In addition, the trips of URT passengers are usually in urban areas, and there are many alternative modes of transportation. Therefore, passengers usually do not choose to wait in case of disruption. The possible behaviors of passengers in section-disruption scenario include rerouting and changing to other transportation modes.

The multi-nomial logit model (MNL) is a classical model for describing discrete choice behavior. Nevertheless, the MNL model only considers the absolute difference of alternative utility, which is known as independence from irrelevant alternation (IIA) property and may lead to a modeling result that does not conform to the actual choice of passengers [12]. To solve this problem, the present study uses the relative utility to calculate the choice probability of each alternative.
We set the shortest travel time between stations $o$ and $d$ before a disruption occurs as $t_{o, d}^{s}$. The shortest travel time of the detour routes after the disruption occurs is denoted as $t_{o, d}^{r}$. Then, the utility of the shortest route before disruption $U_{o, d}^{s}$, that of the shortest detour route after the disruption $U_{o, d}^{r}$, and that of abandoning the URT system to choose other transportation modes $U_{o, d}^{a}$ are expressed as follows:

$$
\begin{aligned}
& U_{o, d}^{s}=-t_{o, d}^{s}+\varepsilon, \\
& U_{o, d}^{r}=-t_{o, d}^{r}+\varepsilon, \\
& U_{o, d}^{a}=-\left(k \cdot t_{o, d}^{s}+t^{p}\right)+\varepsilon,
\end{aligned}
$$

where $\varepsilon$ is a random error that obeys the Gumbel distribution. Because the travel speed in urban ground traffic is usually slower than the underground speed, we use the product of amplification factor $k$ that is greater than 1 , and the travel time of the original URT shortest route $t_{o, d}^{s}$ to describe the travel time of the alternative ground traffic. $t^{p}$ is a constant that indicates the transfer and waiting penalty times resulting from the transfer to the ground traffic.

To avoid the problem caused by the IIA property of the MNL model, we use relative utility to calculate the selection probability of each alternative. The probabilities that a passenger chooses detour route $P_{\text {reroute }}^{o, d}$ and chooses other transportation modes $P_{\text {other }}^{o, d}$ are expressed as follows:

$$
\begin{aligned}
P_{o, d}^{r} & =\frac{\exp \left(\theta \cdot U_{o, d}^{r} / U_{o, d}^{s}\right)}{\exp \left(\theta \cdot U_{o, d}^{r} / U_{o, d}^{s}\right)+\exp \left(\theta \cdot U_{o, d}^{a} / U_{o, d}^{s}\right)}, \\
P_{o, d}^{a} & =\frac{\exp \left(\theta \cdot U_{o, d}^{a} / U_{o, d}^{s}\right)}{\exp \left(\theta \cdot U_{o, d}^{r} / U_{o, d}^{s}\right)+\exp \left(\theta \cdot U_{o, d}^{a} / U_{o, d}^{s}\right)},
\end{aligned}
$$

where $\theta$ is a parameter related to the variance in random error $\varepsilon$ and $U_{o, d}^{r} / U_{o, d}^{s}$ and $U_{o, d}^{a} / U_{o, d}^{s}$ are the relative utilities of detouring and abandoning the URT system, respectively.

\section{Section Vulnerability Indexes}

The effects of a section disruption on the passengers mainly include two aspects. On the one hand, some passengers are delayed by taking detour routes. On the other hand, some 


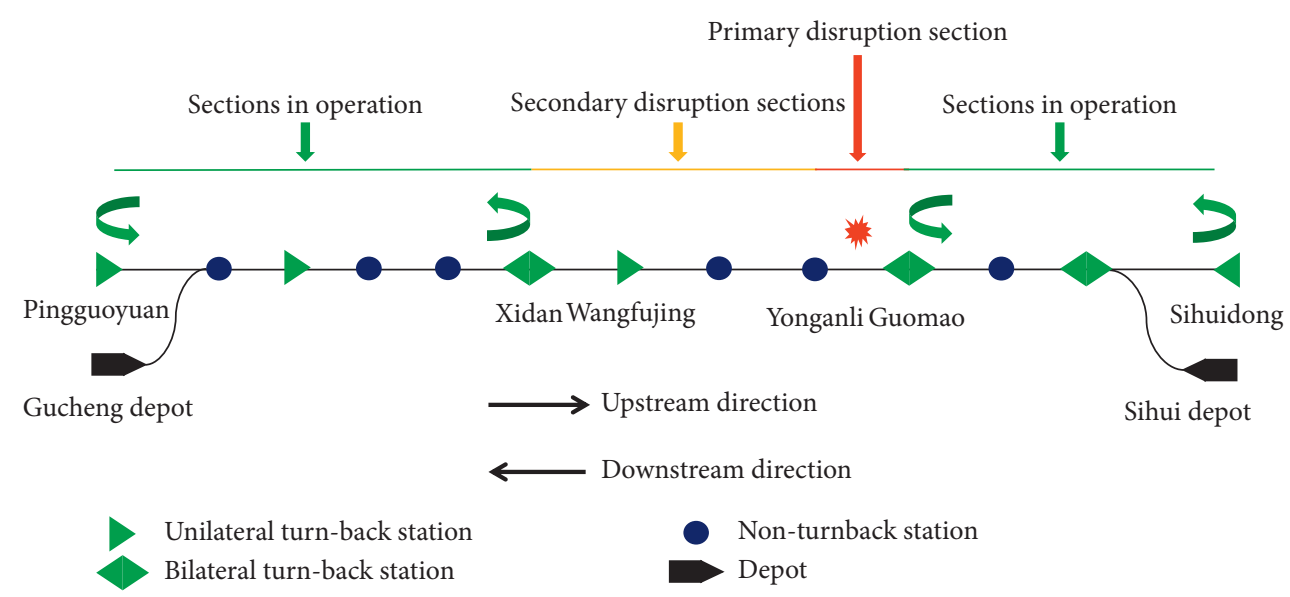

Figure 2: Disruption scenario in Beijing Subway Line 1.

passengers may abandon the URT system because they have no accessible routes after a disruption occurs or the detour routes are very long and unacceptable. Existing URT vulnerability studies pay more attention to passenger accessibility, whereas less attention has been paid to passenger behavior and detour delay. Therefore, by considering the passenger choice behavior, the present study quantitatively analyzes the aforementioned two effects as two measurement indexes of section vulnerability. Then, the vulnerability of the URT network is analyzed by searching the critical section or combination of critical sections with the largest vulnerability indexes.

Detour delay $D^{x}$ and passenger loss $L^{x}$ of a URT network due to section disruption are expressed as follows:

$$
\begin{aligned}
D^{x} & =\sum_{o \in O, d \in D} q_{o, d} \cdot P_{o, d}^{r} \cdot\left(t_{o, d}^{r}-t_{o, d}^{s}\right) \cdot z(x)_{o, d}, \\
L^{x} & =\sum_{o \in O, d \in D} q_{o, d} \cdot P_{o, d}^{a} \cdot t_{o, d}^{s} \cdot z(x)_{o, d},
\end{aligned}
$$

where $q^{o, d}$ denotes the travel demand between station $o$ and $d$ and $x=\left[x_{1}, x_{2}, \ldots, x_{n}\right]$ is the state vector of all sections in the network from 1 to $n$, which indicates whether the sections in the network are disrupted or not. $x_{i}=0$ indicates that section $i$ is in operation, whereas $x_{i}=1$ means section $i$ is disrupted. Similarly, $z(x)_{o, d}=0$ indicates that passengers between $o$ and $d$ are not influenced by state $x$. In contrast, $z(x)_{o, d}=1$ means that passengers between $o$ and $d$ need to find a detour route or choose other transportation modes because of disruption.

$D^{x}$ represents the product of the travel demand and detour delay, that is, the total passenger delay resulting from the detour to other routes. For passengers who abandon the URT during a disruption, measuring the effect of disruption using only the number of passengers who abandon the URT is not logical. For example, for the same passenger, the loss of convenience and economy caused by the inaccessibility of long-distance URT travel is much greater than that caused by short-distance travel inaccessibility. For the URT operating company, the loss of revenue caused by the loss of longdistance passengers is also greater than that caused by the loss of short-distance passengers. Therefore, we use the product of the number of passengers who abandon the URT and their original shortest travel time before disruption $\left(L^{x}\right)$ to measure the effect of passenger-flow loss. To make the vulnerability indexes more intuitive, we use the ratio of $D^{x}$ and $L^{x}$ to $S$ as two measurement indexes of section vulnerability, where the symbol $S$ represents the total passenger travel time of the network under normal conditions.

$$
\begin{aligned}
V_{D}^{x} & =\frac{D^{x}}{S} \\
& =\frac{\sum_{o \in O, d \in D} q_{o, d} \cdot P_{o, d}^{r} \cdot\left(t_{o, d}^{r}-t_{o, d}^{s}\right) \cdot z(x)_{o, d}}{\sum_{o \in O, d \in D} q_{o, d} \cdot t_{o, d}^{s}}, \\
V_{L}^{x} & =\frac{L^{x}}{S} \\
& =\frac{\sum_{o \in O, d \in D} q_{o, d} \cdot P_{o, d}^{a} \cdot t_{o, d}^{s} \cdot z(x)_{o, d}}{\sum_{o \in O, d \in D} q_{o, d} \cdot t_{o, d}^{s}} .
\end{aligned}
$$

\section{Calculation of the Section Vulnerability Indexes}

The section with high vulnerability indexes $V_{D}^{x}$ and $V_{L}^{x}$ is a critical section. Thus, finding the critical sections becomes a multi-objective programming problem with section state vector $x=\left[x_{1}, x_{2}, \ldots, x_{n}\right]$ as the decision variable. Two difficulties are encountered in solving this problem. One is the analysis of the effect of disruption by considering the track layout of the stations and updating the network after a disruption occurs. The other is the efficient calculation of the vulnerability indexes of numerous section combinations. For a large-scale URT network, the vulnerability-index calculation in all section combinations results in a huge amount of computation. By considering the Beijing Subway network with 339 stations and 324 sections as an example, the number of passenger OD pairs exceeds 100,000. Because calculating the shortest route of each OD pair is necessary in the new network after a disruption occurs, this process results in $324 \times 10^{5}$ times shortest route calculation even by considering only the scenario 
of a single-section disruption. When it comes to a multi-section combination disruption scenario, the simultaneous disruption of five sections will result in $C_{324}^{5} \times 105$ times shortest route calculation, which makes the number of shortest route calculations more than $3 \times 10^{23}$ times and impossible to complete within a reasonable time. Therefore, by considering the characteristics of the URT system, we use the correlation among the train routing, travel demand, and rail sections to develop an algorithm and combine it with a heuristic algorithm to compute the indexes for a multi-section-disruption scenario.

4.1. Network Updating. As mentioned in Section 2.1, the disruption of a single section may cause a secondary disruption of the adjacent sections because of the lack of turn-back tracks. The primary and secondary disrupted sections are related by train routings. The URT network can be considered as a set of sections $N=\left[x_{1}, x_{2}, \ldots, x_{n}\right]$. Train routing consists of several sections that satisfy two conditions. First, the two stations at the two ends of these sections must both have tracks for a turn-back operation. Second, these sections require tracks that connect to the depot for trains entering or leaving the depot. All train routings form set $R=\left[R_{1}, R_{2}, \ldots, R_{n}\right]$ in which each train routing is denoted as $R_{m}=\left[x_{1}, x_{2}, \ldots, x_{p}\right]$. When section $x_{i}$ is disrupted, all the train routings that contain $x_{i}$ are disrupted. Then, the sections that do not include any operating train routes are secondarily disrupted. Finally, the set of all primary and secondary disrupted sections is denoted as $x^{\prime}$, and the updated new network $N^{\prime}$ consists of all the operating sections.

In view of a large number of OD pairs in the URT network, which causes an enormous computational burden, we assume that the section disruption only affects the OD pairs whose shortest routes are included in the disrupted section. This assumption is consistent with the reality of a section-disruption scenario and can reduce the computational burden to a certain extent. Variable $z(x)_{o, d}$ indicates whether OD pair $(o, d)$ is affected by the disruption (both primary and secondary).

$z(x)_{o, d}=1$ means that OD pair $(o, d)$ is affected by the disruption. In contrast,

$z(x)_{o, d}=0$ means that OD pair $(o, d)$ is not affected by the disruption. The calculation process for $z(x)_{o, d}$ is described as follows: for any OD pair $o d_{i} \in \mathrm{OD}$, its shortest route $o d_{i}=\left[x_{1}, x_{2}, \ldots, x_{q}\right]$ consists of all the sections in the route. Correspondingly, each section $x_{i}$ corresponds to a set of OD pairs whose shortest routes contain $x_{i}: x_{i}=\left[o d_{1}, o d_{2}, \ldots, o d_{l}\right]$. For all disrupted sections $x_{i}^{\prime} \in x^{\prime}$, the set of affected OD pairs is expressed as

$\mathrm{OD} \prime=x_{1}^{\prime} \cup x_{2}^{\prime} \cup \ldots x_{i}^{\prime}=\left[o d_{1}, o d_{2}, \ldots, o d_{l}\right] \cup\left[o d_{1}, o d_{2}\right.$, $\left.\ldots, o d_{p}\right] \cup \ldots\left[o d_{1}, o d_{2}, \ldots, o d_{q}\right]$. Then, we obtain.

$z(x)_{o, d}=1 \mid o d \in \mathrm{OD}$. The pseudocode of the aforementioned algorithm is listed in Table 1.

4.2. Identification of Critical Sections. Considering the characteristics of a secondary disruption in a URT system, a dominant relationship may exist among some adjacent sections. Calculating the vulnerability indexes only for dominant sections can significantly reduce the search range of the optimal solution. The concepts of equivalent and dominant sections are introduced next.

Definition 1. If the disruption of section $i$ causes disruption of section $j$ and conversely if the disruption of section $j$ causes disruption of section $i$, then sections $i$ and $j$ are equivalent sections.

Definition 2. If the disruption of section $i$ causes disruption of section $j$ while the disruption of section $j$ has no effect on section $i$, then we designate that section $i$ is a dominant section and dominates section $j$.

Figure 3 shows that the disruption of section 1, or 3 will lead to secondary disruption of sections 4,5 , and 6 because of the lack of short-turn trains. Conversely, the disruption of section 4, 5, or 6 does not affect section 1,2, or 3 because the short-turn trains can keep them in operation. In other words, sections 1, 2, and 3 are equivalent to one another and dominate sections 4, 5, and 6. Accordingly, to identify the critical sections, we only need to calculate the vulnerability indexes of section 1 and ignore the others. In order to search dominate section $x^{*}$, we design Algorithm 2 as shown in Table 2. To summarize, the complete calculation process of the URT section vulnerability indexes is shown in Figure 4.

For the single-section-disruption scenario, we can calculate the vulnerability indexes of all the dominant sections, and the section with the maximum indexes is considered the critical one. However, if we consider a multi-section-disruption scenario, a large number of section combinations may be present. Once the number of simultaneous disrupted sections exceeds three, the large number of section combinations will cause an enormous computational burden. In this study, we use the NSGA-II algorithm [13], which is suitable for multi-objective programming problems, to find the critical section combinations. First, the initial population that represents the combination of disrupted sections is generated using the NSGA-II algorithm. Then, objective functions $V_{D}^{x}$ and $V_{L}^{x}$ are calculated using steps 4 and 5 shown in Figure 4. After continuous iteration, several acceptable Pareto solutions are obtained, and each of the solutions represents a critical combination of sections with the greatest effect on the network.

\section{Case Study}

This work considers the Beijing Subway network in 2017 as an example to analyze network vulnerability. The Beijing Subway network, which is a highly networked metropolitan system, consists of 16 lines, 324 sections, and 339 stations. In this case, the passenger-flow data are collected from the automatic fare collection (AFC) system. In terms of parameter setting, we set amplification factor $k$ and transfer penalty time $t^{P}$ in equation (3) to 1.3 and $10 \mathrm{~min}$, respectively. Furthermore, $\theta$ in equations (4) and (5) is set to 4.5 , which means that when the relative utility of Alternative 1 is 1.5 times that of Alternative 2, 90\% of the passengers will choose the former [14]. 
TABLE 1: Pseudo-code of the network-updating algorithm.
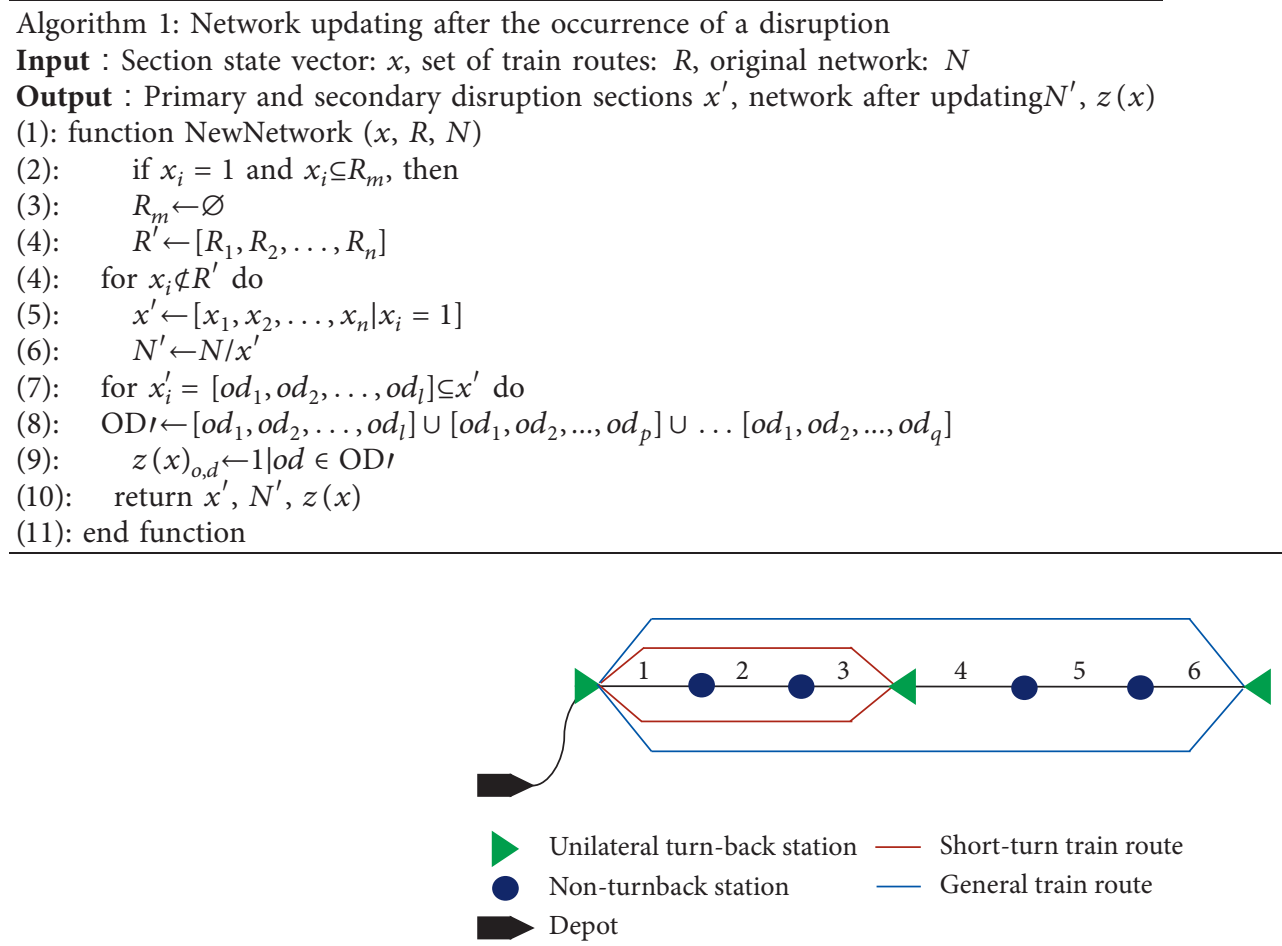

Figure 3: Equivalent and dominant relationship of the sections.

TABle 2: Pseudocode of the dominate section search algorithm.

Algorithm 2: Dominate section search algorithm

Input: Section state vector: $x$, Set of train routes: $R$, Original network: $N$

Output: Set of dominate sections: $x^{*}$

(1): function Dom_Section $(x, R, N)$

(2): for all $x_{i} \in \bar{x}$

(3): $\quad$ do Table 1 function New_Network $\left(x \mid x_{i} \leftarrow 1, R, N\right)$

(4): $\quad$ then $x_{i}^{*} \leftarrow\left[x^{\prime}\right]$

(5): if $x_{j} \subset x_{i}^{*}$ and $x_{i} \not x_{j}^{*}$

(6): $\quad x^{*} \leftarrow x_{i}^{*} / x_{j}$

(7): if $x_{j} \subset x_{i}^{*}$ and $x_{i} \subset x_{j}^{*}$ and $i<j$

(8): $\quad x^{*} \leftarrow x^{*} / x_{j}$

(9): return $x^{*}$

(10): end function

5.1. Single-Section Disruption Case: Identification of Critical Section. The vulnerability indexes of all 324 sections in the Beijing Subway are shown in Figure 5. This shows that only a few sections have a larger effect on the passenger flow in the network and most of the sections have relatively low indexes. As shown by the green dotted line in Figure 5, 70\% of the sections have indexes in the range of $[(0,1.25 \%),(0,6.00 \%)]$ and only $30 \%$ of the sections in the ranger of [ $(1.25 \%, 2.50 \%)$, $(6.00 \%, 12.00 \%)]$. The location of the sections corresponding to the Pareto solution is shown in Figure 6. The disruption of section (1), i.e., Taoranting-Caishikou (TRT-CSK) in Subway Line 4 causes the largest passenger loss $V_{L}^{x}$. Because of the lack of turn-back tracks, the disruption of section (1) leads to the secondary disruption of the four adjacent sections. Then, the five sections from Gongyixiqiao (GYXQ) to Caishikou (CSK) are all disrupted, which forces a large number of passengers to abandon the URT because of the lack of detour routes. The disruption of section (4), i.e., Chaoyangmen-Dongdaqiao (CYM-DDQ) in Subway Line 6 causes the largest detour delay $V_{D}^{x}$ because passengers can detour to Subway Line 1, which is parallel to Subway Line 6. The disruption of sections (2) and (3) not only causes a large detour delay but also affects the accessibility of a large number of passengers, which also greatly affects the passenger flow in the network.

Vulnerability indexes $V_{D}^{x}$ and $V_{L}^{x}$ in each section in the network are shown in Figures 7(a) and 7(b), respectively, which show that the sections with large detour delay $V_{D}^{x}$ are usually located in the dense area in the network or in a loop line because the passengers have an alternative route to detour once this type of section is disrupted. Correspondingly, the sections in the suburban radial lines usually suffer from a relatively lower detour delay because of the lack of alternative routes. In contrast, the sections with large 


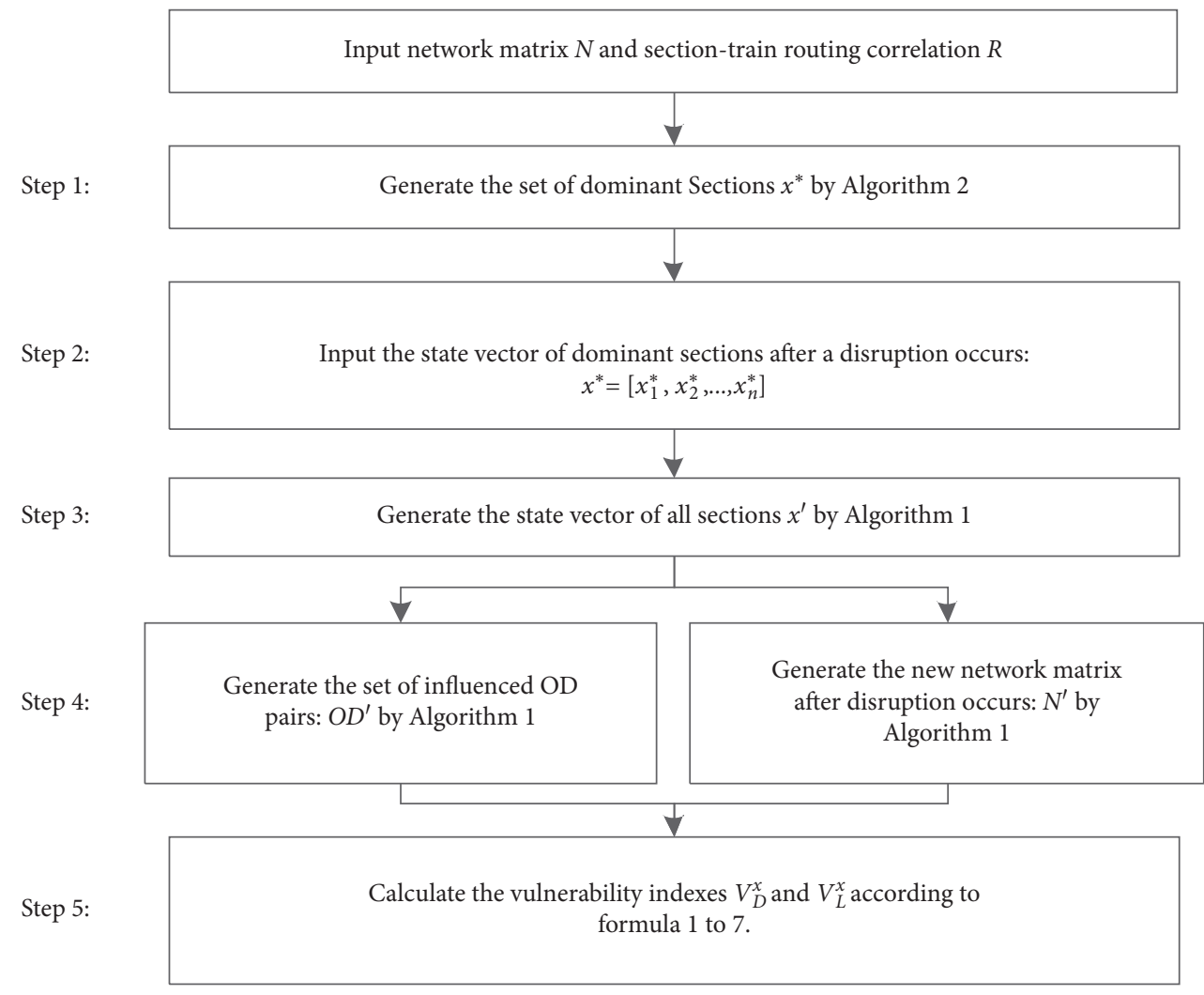

FIGURE 4: Calculation process of the URT section vulnerability indexes.

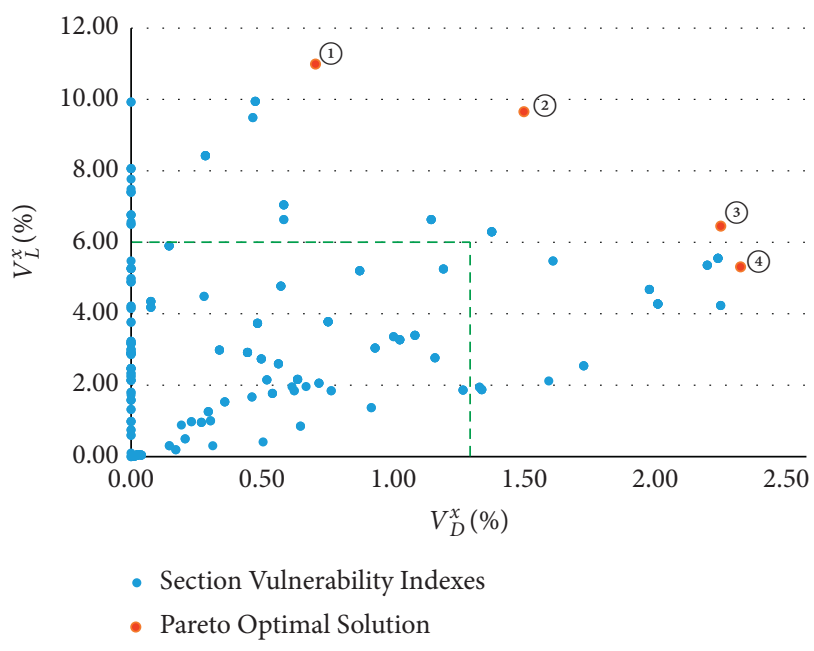

FIgURE 5: Distribution of section vulnerability indexes.

passenger loss $V_{L}^{x}$ are usually located at the junction of the radial and loop lines. Once these sections are disrupted, a large number of passengers will abandon the URT because no alternative route is available.

\subsection{Multi-Section Disruption Case: Identification of Critical} Section Combination. Once the URT network is intentionally attacked, simultaneous disruptions of multiple sections may occur. Therefore, exploring the characteristics of a multi-section combined disruption is necessary to analyze

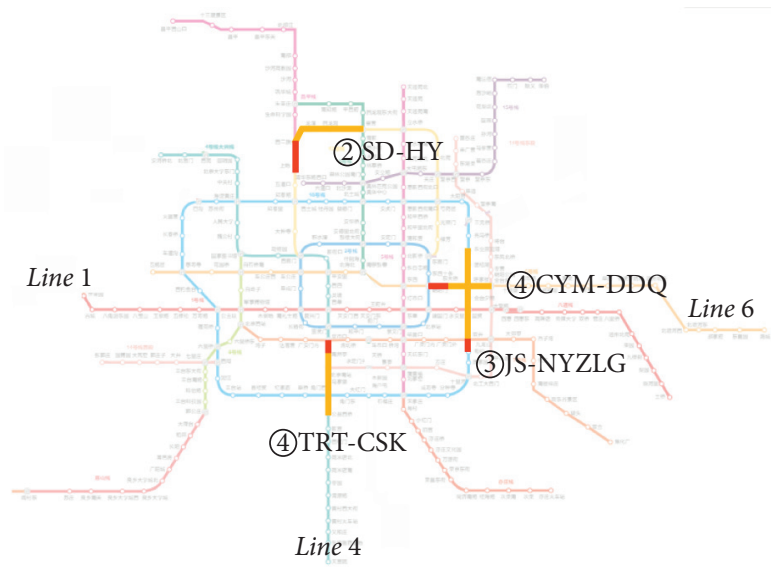

Figure 6: Location of the sections corresponding to the Pareto solution.

the network vulnerability. Section 4 presented that an enormous number of section combinations will result in a serious computational burden. To solve this problem, we propose the concept of "dominant section" to narrow the search range of the critical section combination. We also adopt the NSGA-II algorithm to improve computational efficiency.

The Pareto solutions of the section combination vulnerability index of two sections under a simultaneous disruption scenario are listed in Table 3. Index Diff denotes the difference between the multi-section combination vulnerability indexes and the sum of each single-section 


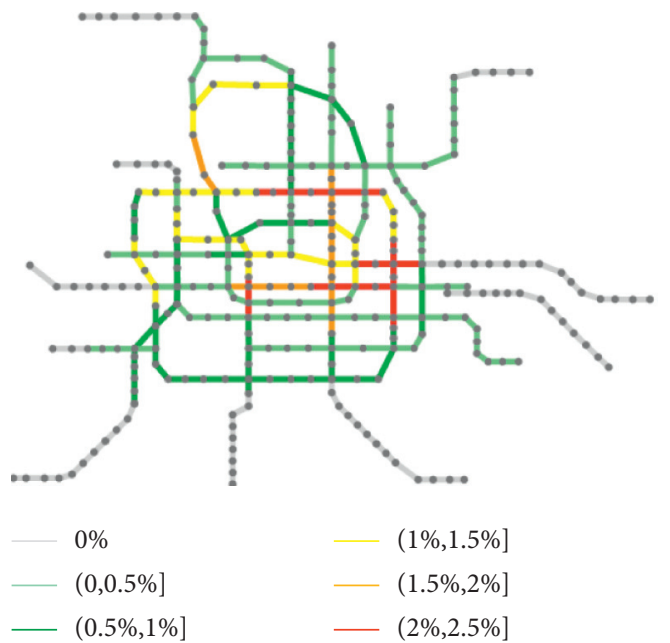

(a)

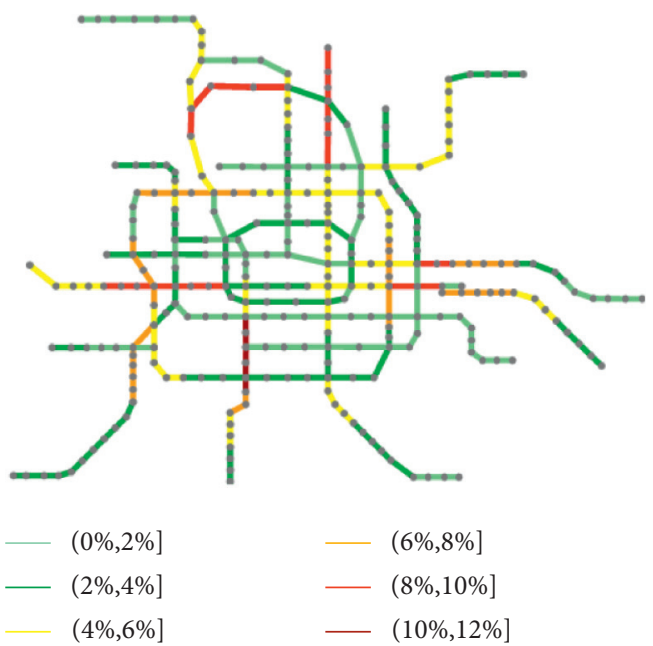

(b)

Figure 7: Vulnerability indexes in each section in the Beijing Subway network: (a) detour delay $V_{D}^{x}$ and (b) passenger loss $V_{L}^{x}$.

TABLE 3: Pareto solutions of section combinations in a simultaneous two-section disruption

\begin{tabular}{|c|c|c|c|c|c|c|c|c|c|}
\hline \multicolumn{3}{|c|}{ Vulnerability indexes of a single section } & \multicolumn{3}{|c|}{$\begin{array}{l}\text { Vulnerability indexes of a single } \\
\text { section }\end{array}$} & \multicolumn{2}{|c|}{$\begin{array}{l}\text { Vulnerability } \\
\text { indexes of the } \\
\text { section } \\
\text { combination }\end{array}$} & \multicolumn{2}{|c|}{$\begin{array}{c}\text { Diff }=V^{1,2}-V^{1}- \\
V^{2}\end{array}$} \\
\hline Section 1 & $V_{D}^{1}$ & $V_{L}^{1}$ & Section 2 & $V_{D}^{2}$ & $V_{L}^{2}$ & $V_{D}^{1,2}$ & $V_{L}^{1,2}$ & $\operatorname{Diff}_{D}$ & $\operatorname{Diff}_{L}$ \\
\hline BBS-YQL & $0.00 \%$ & $5.27 \%$ & SH-SHD & $0.30 \%$ & $0.30 \%$ & $1.09 \%$ & $20.13 \%$ & $0.79 \%$ & $14.57 \%$ \\
\hline GZF-JSBWG & $0.46 \%$ & $9.94 \%$ & SH-SHD & $0.30 \%$ & $0.30 \%$ & $1.09 \%$ & $20.10 \%$ & $0.33 \%$ & $9.86 \%$ \\
\hline GZF-JSBWG & $0.46 \%$ & $9.94 \%$ & TRT-CSK & $0.68 \%$ & $10.99 \%$ & $1.09 \%$ & $20.37 \%$ & $-0.06 \%$ & $-0.57 \%$ \\
\hline WFJ-DD & $2.13 \%$ & $5.36 \%$ & JS-SJ & $2.18 \%$ & $6.46 \%$ & $4.31 \%$ & $12.62 \%$ & $0.00 \%$ & $0.81 \%$ \\
\hline WFJ-DD & $2.13 \%$ & $5.36 \%$ & TYG-SYJ & $2.17 \%$ & $5.55 \%$ & $4.62 \%$ & $11.35 \%$ & $0.32 \%$ & $0.44 \%$ \\
\hline WFJ-DD & $2.13 \%$ & $5.36 \%$ & XEQ-SD & $1.45 \%$ & $9.66 \%$ & $3.58 \%$ & $14.97 \%$ & $-0.01 \%$ & $-0.05 \%$ \\
\hline GM-DWL & $0.45 \%$ & $9.49 \%$ & TRT-CSK & $0.68 \%$ & $10.99 \%$ & $1.09 \%$ & $20.16 \%$ & $-0.04 \%$ & $-0.32 \%$ \\
\hline TRT-CSK & $0.68 \%$ & $10.99 \%$ & CYM-DDQ & $2.26 \%$ & $5.32 \%$ & $2.89 \%$ & $16.17 \%$ & $-0.05 \%$ & $-0.15 \%$ \\
\hline TRT-CSK & $0.68 \%$ & $10.99 \%$ & JTL-SLP & $0.00 \%$ & $9.93 \%$ & $0.67 \%$ & $20.66 \%$ & $-0.02 \%$ & $-0.26 \%$ \\
\hline TRT-CSK & $0.68 \%$ & $10.99 \%$ & JS-SJ & $2.18 \%$ & $6.46 \%$ & $2.81 \%$ & $17.21 \%$ & $-0.05 \%$ & $-0.23 \%$ \\
\hline TRT-CSK & $0.68 \%$ & $10.99 \%$ & TYG-SYJ & $2.17 \%$ & $5.55 \%$ & $2.86 \%$ & $16.49 \%$ & $0.00 \%$ & $-0.05 \%$ \\
\hline TRT-CSK & $0.68 \%$ & $10.99 \%$ & XEQ-SD & $1.45 \%$ & $9.66 \%$ & $1.97 \%$ & $19.99 \%$ & $-0.17 \%$ & $-0.67 \%$ \\
\hline YHG-HPLBJ & $1.95 \%$ & $4.27 \%$ & JS-SJ & $2.18 \%$ & $6.46 \%$ & $4.30 \%$ & $12.68 \%$ & $0.17 \%$ & $1.95 \%$ \\
\hline CYM-DDQ & $2.26 \%$ & $5.32 \%$ & XEQ-SD & $1.45 \%$ & $9.66 \%$ & $3.65 \%$ & $14.88 \%$ & $-0.06 \%$ & $-0.10 \%$ \\
\hline JS-SJ & $2.18 \%$ & $6.46 \%$ & XEQ-SD & $1.45 \%$ & $9.66 \%$ & $3.52 \%$ & $15.79 \%$ & $-0.11 \%$ & $-0.33 \%$ \\
\hline TYG-SYJ & $2.17 \%$ & $5.55 \%$ & XEQ-SD & $1.45 \%$ & $9.66 \%$ & $3.23 \%$ & $15.99 \%$ & $-0.40 \%$ & $0.78 \%$ \\
\hline
\end{tabular}

vulnerability indexes. We can see that the effect of the multisection combination disruption on the passenger flow is not simply a superposition of the effect of each single-section disruption. For example, the simultaneous disruptions of sections Babaoshan-Yuquanlu (BBS-YQL) and Sihui-Sihuidong (SH-SHD) results in a $14.57 \%$ higher passenger loss than the summation of the two single-section disruptions because the simultaneous disruptions of the two sections lead to failure of entire Subway Line 1, which causes a large number of passengers to have no accessible routes. However, because overlap is present between the passenger flow serviced by sections TRT-CSK and Xierqi-Shangdi (XEQ-SD), the vulnerability indexes of this section combination are smaller than the summation of the two single sections. Therefore, the vulnerability indexes of a multisection combination represent the result of the interaction between the location of the sections and the passenger-flow distribution in the network, which is highly significant for the analysis of the URT network vulnerability.

We conducted an experiment using different numbers of section combination disruptions. Maximum $V_{D}^{x}$ and $V_{L}^{x}$ under different numbers of sections are shown in Figures 8 (a) and $8(\mathrm{~b})$, respectively. Vulnerability indexes $V_{D}^{x}$ and $V_{L}^{x}$ dramatically change with the number of disrupted sections when the number is less than 15 . When the number of disrupted sections is more than 15 , the changes in the indexes are relatively stable. Therefore, we adopt a larger sampling interval when the number of disrupted sections is greater than 15 to reduce the amount of calculation. Figure 8(a) shows that the variation curve of detour delay $V_{D}^{x}$ with the number of disrupted section is close to a paracurve, which reaches a maximum value when seven sections are 


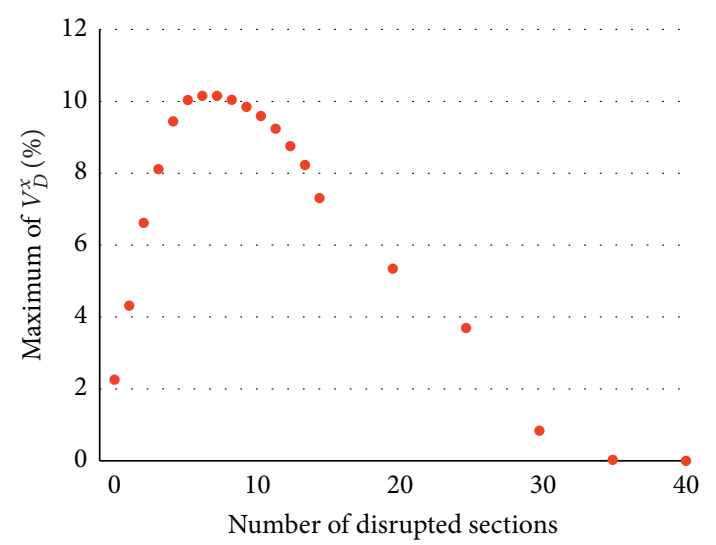

(a)

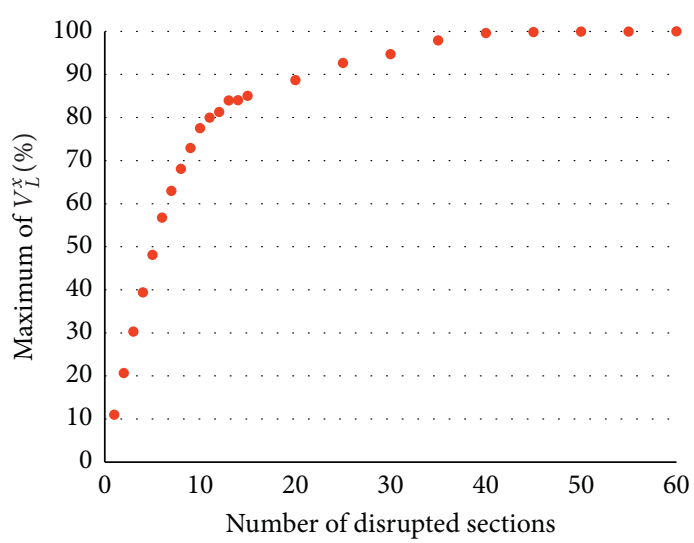

(b)

FiguRe 8: Maximum vulnerability indexes under different numbers of disrupted sections: (a) maximum detour delay $V_{D}^{x}$ and (b) maximum passenger loss $V_{L}^{x}$.

simultaneously disrupted and then reduces to 0 with the increase in the number of disrupted sections. This result occurs because with the increase in the number of disruptions, the network structure is damaged and the number of passenger detour routes continuously decreases until no detour route is available. This phenomenon is also reflected by passenger loss $V_{L}^{x}$. Figure $8(\mathrm{~b})$ shows that the variation curve of passenger loss $V_{L}^{x}$ with the number of disrupted sections closely resembles a logarithmic curve. When the number of disrupted sections is less than $10, V_{L}^{x}$ sharply increases with the increase in the number of disruptions and then gradually approaches $100 \%$. Obviously, a few critical sections or critical section combinations will cause a significant effect on the capacity of the URT network, and disruptions even in only $3 \%$ of the sections can lead to $80 \%$ passenger-flow loss.

\section{Conclusions}

This study has analyzed the vulnerability of a URT network by identifying the critical section and combination of sections. Because the modeling of disruption scenarios and passenger behavior in existing URT vulnerability studies are not sufficiently realistic, we consider the station track layout and model the passenger choice behavior during disruption using a revised MNL model. Then, we propose two section vulnerability indexes that measure the effect of a URT section disruption from two aspects: detour delay and passenger-flow loss due to travel inaccessibility. To narrow the scope of the multi-section combinations, we propose the concept of "dominant section" and combine it with the NSGA-II algorithm to identify the critical section combinations. Finally, a case study of the Beijing Subway network is conducted to verify the method.

The results of the case study show that section disruptions that occur at different locations in the URT network cause varying degrees of passenger-flow delay and losses. The disruption consequences are affected by the track layout in each station and the distribution of the passenger flow. When a multi-section disruption occurs, the effect is not a simple superposition of each single-section disruption. Generally, even a few critical sections can greatly affect the passenger flow of the URT network, and disruption of even only $3 \%$ of the sections can lead to $80 \%$ passenger-flow loss, which reflects the high vulnerability of the URT network. Our method can provide support for the evaluation of URT network performance. Because this study only considers the section disruption and ignores other incidents such as long delays in the sections, we will analyze more incidents and consider the probability of disruption occurrence into account in future research.

\section{Data Availability}

All the related data are included within this article.

\section{Conflicts of Interest}

The authors declare that they have no conflicts of interest.

\section{Acknowledgments}

This work was supported by the Beijing Natural Science Foundation (9202012).

\section{References}

[1] L. G. Mattsson and E. Jenelius, "Vulnerability and resilience of transportation systems-a discussion of recent research," Transportation Research Part A: Policy and Practice, vol. 81, pp. 16-34, 2015.

[2] G. Bankoff, G. Frek, and D. Hilhorst, Mapping Vulnerability: Disasters, Development and People, R, London, 2013.

[3] K. Berdica, "An introduction to road vulnerability: what has been done, is done and should be done," Transport Policy, vol. 9, no. 2, pp. 117-127, 2002.

[4] U. Demšar, O. Špatenková, and K. Virrantaus, "Identifying critical locations in a spatial network with graph theory," Transactions in GIS, vol. 12, no. 1, pp. 61-82, 2008.

[5] J. Zhang, S. Wang, and X. Wang, "Comparison analysis on vulnerability of metro networks based on complex network," 
Physica A: Statistical Mechanics and Its Applications, vol. 496, pp. 72-78, 2018.

[6] A. Nicholson and Z. Du, "Degradable transportation systems: an integrated equilibrium model," Transportation Research Part B: Methodological, vol. 31, no. 3, pp. 209-223, 1997.

[7] E. Jenelius, "Network structure and travel patterns: explaining the geographical disparities of road network vulnerability," Journal of Transport Geography, vol. 17, no. 3, pp. 234-244, 2009.

[8] D. Z. W. Wang, H. Liu, W. Y. Szeto, and H. F. Chow, "Identification of critical combination of vulnerable links in transportation networks-a global optimisation approach," Transportmetrica: Transportation Science, vol. 12, no. 4, pp. 346-365, 2016.

[9] X. Xu, A. Chen, and C. Yang, "An optimization approach for deriving upper and lower bounds of transportation network vulnerability under simultaneous disruptions of multiple links," Transportation Research Part C: Emerging Technologies, vol. 94, pp. 338-353, 2018.

[10] H. Shimamoto, F. Kurauchi, J. D. Schmöcker, and M. G. H Bell, "Evaluating critical lines and stations considering the impact of the consequence using transit assignment model-case study of London's underground network," Journal of Advanced Transportation, vol. 42, no. 3, pp. 291-310, 2008.

[11] D. Sun and S. Guan, "Measuring vulnerability of urban metro network from line operation perspective," Transportation Research Part A: Policy and Practice, vol. 94, pp. 348-359, 2016.

[12] J. Zhang, H. Timmermans, A. Borgers, and D. A Wang, "Modeling traveler choice behavior using the concepts of relative utility and relative interest," Transportation Research Part B: Methodological, vol. 38, no. 3, pp. 0-234, 2004.

[13] K. Deb, A. Pratap, S. Agarwal, and T. Meyarivan, "A fast and elitist multiobjective genetic algorithm: nsga-II," IEEE Transactions on Evolutionary Computation, vol. 6, no. 2, pp. 182-197, 2002.

[14] E. van der Hurk, Passengers, information, and disruptions, $\mathrm{PhD}$ dissertation, Erasmus University Rotterdam, Rotterdam, Netherlands, 2015. 